\title{
Identifying Cancer Subjects With Acute Respiratory Failure at High Risk for Intubation and Mechanical Ventilation
}

\author{
Virginie Lemiale MD, Jérôme Lambert MD, Emmanuel Canet MD, Djamel Mokart MD, \\ Frederic Pène MD PhD, Antoine Rabbat MD, Achille Kouatchet MD, François Vincent MD, \\ Fabrice Bruneel MD, Didier Gruson MD, Sylvie Chevret MD PhD, and Elie Azoulay MD PhD, \\ a Groupe de Recherche Respiratoire en Réanimation Onco-Hématologique Study
}

\begin{abstract}
BACKGROUND: We sought to identify risk factors for mechanical ventilation in patients with malignancies and acute respiratory failure (ARF). METHODS: We analyzed data from a previous randomized controlled trial in which nonintubated oncology and hematology subjects with ARF were randomized to early bronchoalveolar lavage or routine care in 16 ICUs in France. Consecutive patients with malignancies were admitted to the ICU for ARF in 2005 and 2006 with no intervention. RESULTS: During the study period, 219 patients were admitted to the ICU for ARF, and 8 patients were not included due to a nonintubation order. Data on the underlying disease, pulmonary involvement, and extrapulmonary organ dysfunctions were recorded at admission in the 211 remaining subjects. Ventilatory support included oxygen only (49 subjects), noninvasive ventilation (NIV) only (81 subjects), NIV followed by invasive mechanical ventilation (49 subjects), and firstline invasive mechanical ventilation ( 32 subjects). The 81 subjects who required invasive mechanical ventilation were compared with the 130 subjects who remained on oxygen or NIV. Factors associated with invasive mechanical ventilation by multivariate analysis were the oxygen flow required at ICU admission, the number of quadrants involved on chest $x$-ray, and hemodynamic dysfunction. Mortality rates for subjects who had NIV failure were $65.3 \%$ compared with $50 \%$ for subjects who were first-line intubated $(P=.34)$. CONCLUSIONS: In cancer patients with ARF, hypoxemia, extent of pulmonary infiltration on chest $\mathrm{x}$-ray, or hemodynamic dysfunction are risk factors for invasive mechanical ventilation. Mortality was not significantly different between NIV failure and first-line intubation. Key words: respiratory failure; noninvasive ventilation; immunosuppression; outcome; cancer mechanical ventilation. [Respir Care 2014;59(10):1517-1523. (C) 2014 Daedalus Enterprises]
\end{abstract}

\section{Introduction}

Acute respiratory failure (ARF) is frequent in patients with malignancies. ${ }^{1}$ Indeed, respiratory events occur in $1 \%$ (oncology) to $20 \%$ (hematology) of patients, reaching $40 \%$ in neutropenic patients and bone marrow transplant recipients. ${ }^{2}$ Half of the patients with respiratory complications will require ICU admission for ARF or associated organ dysfunction. ${ }^{1-3}$ As shown in earlier studies, ${ }^{4,5}$ when oncol-

\footnotetext{
Hospital, Versailles, France. Dr Gruson is affiliated with Pellegrin Teaching Hospital, Bordeaux, France.

Supplementary material related to this paper is available at http:// www.rcjournal.com.

This work was supported by Assistance Publique-Hôpitaux de Paris grant AOM 04.139 and the French Society for Intensive Care.
}

Drs Lemiale, Canet, and Azoulay are affiliated with the ICU, and Drs Lambert and Chevret are affiliated with the Biostatistics Department, Saint Louis Teaching Hospital, Paris, France. Dr Mokart is affiliated with CU, Institut Paoli Calmettes, Marseilles, France. Dr Pene is affiliated affiliated with the ICU, Hotel Dieu Teaching Hospital, Paris, France. Dr Kouatchet is affiliated with the ICU, Angers Teaching Hospital, Angers, France. Dr Vincent is affiliated with the ICU, Avicenne Teaching Hospital, Bobigny, France. Dr Bruneel is affiliated with the ICU, Mignot 
ogy and hematology patients become critically ill, in-hospital mortality is up to $50 \%$. Mortality was highest in patients needing intubation and invasive mechanical ventilation, particularly when started after the first $3 \mathrm{~d}$ in the ICU. $2,3,5,6$ In that setting, mortality may reach $60-90 \% .^{7}$ Other factors associated with mortality were worse performance status on the Eastern Cooperative Oncology Group/World Health Organization scale, ${ }^{8}$ allogeneic stem cell transplantation, high organ failure scores, increased organ dysfunction throughout the ICU stay, as well as diagnosis of invasive pulmonary aspergillosis., , $^{2,9-13} \mathrm{ARF}$ may be related to various etiologies, and failure to identify the cause is associated with increased mortality., ${ }^{2,13-17}$ Invasive procedures such as bronchoalveolar lavage (BAL) or lung biopsy may be required to identify the cause but may also increase the risk of requiring invasive mechanical ventilation. ${ }^{15,18-21}$

Noninvasive ventilation (NIV) is an effective alternative to invasive mechanical ventilation in patients with malignancies and ARF. ${ }^{22}$ Studies have shown that NIV may avoid intubation and improve survival rates. ${ }^{22-25}$ Thus, mortality was lower in patients who responded to NIV.22,24 However, failure of NIV requiring second-line invasive mechanical ventilation was associated with high mortality rates ${ }^{26,27}$ and, in one study, with increased mortality compared with first-line invasive mechanical ventilation. ${ }^{1}$ However, most of these studies were retrospective, and they reported the use of prolonged NIV in subjects with extrapulmonary organ dysfunction who were subsequently intubated. ${ }^{27}$ If cases are identified at admission, patients at risk for intubation may help clinicians guide initial ventilatory strategies without losing a chance for optimal management. Hence, we began the present study with the objective of identifying factors present at ICU admission that were associated with a need for invasive mechanical ventilation.

\section{Methods}

\section{Subjects and Study Design}

This study is a post hoc analysis of data from a multicenter randomized trial conducted in 16 medical or surgical ICUs in France in 2005-2006 (Institutional Review Board number 3705, December 5, 2005). The detailed protocol and subject characteristics have been reported elsewhere. ${ }^{19}$ The appropriate ethics committee approved

Correspondence: Virginie Lemiale MD, AP-HP, Hôpital Saint-Louis, Medical ICU, 1 avenue Claude Vellefaux, 75010 Paris, France. E-mail: virginie.lemiale@sls.aphp.fr.

DOI: $10.4187 /$ respcare.02693

\section{QUICK LOOK}

\section{Current knowledge}

Acute respiratory failure is a frequent complication in patients with malignancies. The requirement for mechanical ventilation in hematology patients and those after bone marrow transplant is associated with a mortality rate approaching $50 \%$.

\section{What this paper contributes to our knowledge}

Cancer patients with acute respiratory failure and hemodynamic failure at ICU admission were at risk for requiring invasive mechanical ventilation. Mortality rate was similar between patients who failed noninvasive ventilation and those who received invasive ventilation primarily.

the research protocol in March 2005. We obtained written informed consent from all patients before study inclusion.

In brief, this randomized trial included patients with hematological or solid malignancies who were admitted to the ICU for ARF, which was defined as an oxygen saturation of $<90 \%$ or $\mathrm{P}_{\mathrm{aO}_{2}}$ of $<60 \mathrm{~mm} \mathrm{Hg}$ on room air combined with severe dyspnea at rest with inability to speak in sentences, breathing frequency of $>30$ breaths/min, or clinical signs of respiratory distress. Patients with contraindications to bronchoscopy and BAL (coma, shock, or arterial oxygen saturation $<90 \%$ while breathing oxygen through an air-entrainment mask) were not included. We did not include patients with cardiogenic pulmonary edema, ARF due to known causes, invasive mechanical ventilation before ICU admission, treatment-limitation decisions, pregnancy or lactation, or previous enrollment in this or another interventional trial. All subjects underwent noninvasive diagnostic tests. Subjects were allocated at random to routine early BAL or to BAL only in the event of intubation or failure to identify the cause of ARF. BAL was performed in a segmental bronchus in the middle lobe or in the lung segment exhibiting the most severe abnormalities by chest radiograph or computed tomography, using four $50-\mathrm{mL}$ aliquots of warm sterile isotonic saline solution. The bronchoscope was inserted transnasally or transorally into the trachea after instillation of a topical nasal and pharyngeal anesthetic. Different diagnostic tests were then performed: pathology, Grocott stain, specific immunofluorescence test, and a polymerase chain reaction technique were used to detect $P$. jiroveci; polymerase chain reaction techniques or viral antigen were used to detect viruses; and Gram stain, Ziehl-Neelsen stain, and culture were used to detect bacteria and fungi. ${ }^{19}$ (See the supplementary materials at http://www.rcjournal.com.) All study data were collected prospectively during the first $28 \mathrm{~d}$ 


\section{Identifying Cancer Subjects at Risk for Intubation and Mechanical Ventilation}

after randomization. All randomized subjects except those with a no-intubation order were included in this post hoc study.

The decision to intubate was made by the physician in charge of the patient. The criteria for intubation were as follows: failure to maintain $\mathrm{P}_{\mathrm{aO}} / \mathrm{F}_{\mathrm{IO}_{2}}>85$, the development of conditions necessitating endotracheal intubation to protect the airway (a seizure disorder or severe encephalopathy with a score on the Glasgow coma scale of $\leq 8$ ), the development of copious tracheal secretions, an increase in the $\mathrm{P}_{\mathrm{aCO}}{ }_{2}$ accompanied by a pH of 7.30 or less, agitation requiring sedation, or hemodynamic instability (defined as a systolic blood pressure of $<70 \mathrm{~mm} \mathrm{Hg}$, need for vasopressors, or evidence on electrocardiography of ischemia or clinically important ventricular arrhythmia).

\section{Study Variables}

At admission, data that are presented in Table 1 were prospectively collected. Each day, the modified Logistic Organ Dysfunction Score (LODS), ${ }^{28}$ based on hepatic, neurologic, kidney, and cardiovascular failure, was assessed. Hematologic failure and ARF were required for study inclusion and consequently were not included in the modified LODS (Logistic Organ Dysfunction System). The type of malignancy, neutropenia, allogeneic bone marrow transplantation, and time from respiratory symptom onset to ICU admission were recorded, as well as the cause of ARF, diagnostic procedures used (noninvasive tests only or with BAL), and chest radiograph features on the first ICU day. Allocated group (BAL or noninvasive procedure) was also analyzed. In intubated subjects, we recorded the time from BAL to intubation and the use of NIV before intubation. NIV started on the same day as invasive mechanical ventilation for a duration not exceeding $1 \mathrm{~h}$ was considered a pre-oxygenation method and not a treatment procedure. All intubations during ICU stay were included in the analysis.

\section{Statistical Analysis}

All data are presented as the median (25th-75th percentile) for quantitative variables and as frequencies (percentage) for qualitative variables. Organ dysfunction was assessed by dichotomizing the LODS at day $1($ LODS $=0$ or LODS $>0$ ). Baseline characteristics were compared across the groups of intubated and nonintubated subjects by using the Wilcoxon rank-sum test or the Fisher exact test. Variables statistically associated $(P<.05)$ were then included in a multivariable logistic regression model. Results of the logistic regression are presented with odds ratio and $95 \%$ CI. To take into account a possible center effect in the rate of intubation, a random center effect (random intercept) was included in the logistic model. All centers that included $<5$ patients were aggregated in one artificial center. Missing baseline covariates were handled via multivariate imputation by chained equations. All statistical analyses were performed using R 2.13.1 software. In the multivariate analysis, withdrawal of life-sustaining treatment was assessed. Those patients were excluded from the analysis presented. In a second analysis (data not shown), subjects were considered as intubated on day of death, and then in a third analysis (data not shown), subjects were considered not intubated on day of death.

\section{Results}

Among the 314 cancer patients admitted for ARF in the 16 participating ICUs between September 2005 and November 2007, 220 were randomized. Informed consent was withdrawn by one patient, leaving 219 patients to participate in the study. Of the 219 included subjects, 8 were not intubated because of end-of-life decision and were not analyzed (Fig. 1).

Of the 211 subjects, $69.6 \%$ were male, the median age was $62(49-70) \mathrm{y}$, and the median body mass index was 24 $\mathrm{kg} / \mathrm{m}^{2} .{ }^{21-27}$ Most of the subjects (83.8\%) had hematological malignancies. Neutropenia was a feature on ICU admission in $36 \%$ of subjects. Half of the subjects $(50.2 \%)$ had comorbidity: $38.3 \%$ had cardiovascular disease, $15.6 \%$ had chronic respiratory failure, $5.2 \%$ had chronic renal failure, and $10.4 \%$ had diabetes. Of the 211 remaining subjects, $81(38.3 \%)$ received invasive mechanical ventilation during the ICU stay and were compared with 130 subjects who never received invasive mechanical ventilation (Fig. 2).

Table 1 illustrates the univariate analysis of risk factors associated with invasive mechanical ventilation. Subjects who required invasive mechanical ventilation were admitted after longer stays on the ward, had greater severity of respiratory failure with higher oxygen requirements, and had a larger number of lung quadrants with radiographic infiltrates. Furthermore, subjects with a higher number of extrapulmonary organ dysfunction and those with an infection were at greater risk for invasive mechanical ventilation. The risk of invasive mechanical ventilation was not influenced by the type of malignancy. The rate of invasive mechanical ventilation use was not significantly different across the study centers.

Of the 105 subjects who were not intubated at the time of bronchoscopy and BAL, $71(67.6 \%)$ received the procedure with oxygen only, and $34(32.4 \%)$ received NIV to secure the procedure (including 19 subjects who received NIV during the procedure and 15 who received NIV just before and just after the procedure). Overall, bronchoscopy and BAL did not significantly affect subjects' respiratory status (ie, breathing frequency and oxygen requirement within $24 \mathrm{~h}$ after the procedure). However, it should 


\section{Identifying Cancer Subjects at Risk for Intubation and Mechanical Ventilation}

Table 1. Univariate Analysis of Characteristics on Admission in Intubated and Nonintubated Subjects

\begin{tabular}{|c|c|c|c|}
\hline Variable & Nonintubated $(n=130)$ & Intubated $(n=81)$ & $P$ \\
\hline Age, median (range), y & $60(47-70)$ & $63(52-69)$ & .22 \\
\hline Body mass index, median (range), $\mathrm{kg} / \mathrm{m}^{2}$ & $24(22-26)$ & $24(21-27)$ & .61 \\
\hline \multicolumn{4}{|l|}{ Comorbidities, $n(\%)$} \\
\hline Cardiovascular comorbidity & $54(41.5)$ & $27(33.3)$ & .24 \\
\hline COPD & $22(16.9)$ & $11(13.6)$ & .56 \\
\hline Chronic renal failure & $7(5.4)$ & $4(4.9)$ & .85 \\
\hline Diabetes & $13(10)$ & $9(11.1)$ & .80 \\
\hline \multicolumn{4}{|l|}{ Underlying malignancy, $n(\%)$} \\
\hline Hematological & $109(83.8)$ & $68(84)$ & \\
\hline Lung cancer & $8(6.2)$ & $4(4.9)$ & .91 \\
\hline Other malignancy & $13(10)$ & $9(11.1)$ & \\
\hline \multicolumn{4}{|l|}{ Underlying hematologic malignancy, $n(\%)$} \\
\hline Acute leukemia & $47(36.2)$ & $20(24.7)$ & .34 \\
\hline Non-Hodgkin lymphoma & $23(17.7)$ & $18(22.2)$ & \\
\hline Myeloma & $15(11.5)$ & $9(11.1)$ & \\
\hline Other hematologic malignancy & $45(34.6)$ & $34(42)$ & \\
\hline Allogeneic bone marrow transplantation, $n(\%)$ & $16(14.7)$ & $12(17.6)$ & .67 \\
\hline Neutropenia at admission, $n(\%)$ & $32(25.8)$ & $26(32.9)$ & .34 \\
\hline \multicolumn{4}{|l|}{ Pulmonary examination, $n(\%)$} \\
\hline Crackles & $46(36.5)$ & $32(41.6)$ & .10 \\
\hline Squeaks & $17(13.5)$ & $16(20.8)$ & \\
\hline Sputum production, $n(\%)$ & $30(23.1)$ & $13(16.2)$ & .29 \\
\hline \multicolumn{4}{|l|}{ Cause of acute respiratory disease, $n(\%)$} \\
\hline Infection & $49(37.7)$ & $24(29.6)$ & .05 \\
\hline Cardiogenic edema & $7(5.4)$ & $3(3.7)$ & \\
\hline Opportunistic infection & $27(20.8)$ & $32(39.5)$ & \\
\hline Miscellaneous reasons & $21(16.2)$ & $7(8.6)$ & \\
\hline Undetermined & $26(20)$ & $15(18.5)$ & \\
\hline Time from symptom onset to ICU admission, median (range), d & $2.5(0-5.2)$ & $2(1-6)$ & .3 \\
\hline Time from hospital admission to ICU admission, median (range), d & $1(0-9.2)$ & $4(0.5-17.5)$ & .05 \\
\hline $\mathrm{pH}$ at admission, median (range) & $7.44(7.39-7.48)$ & $7.45(7.40-7.49)$ & .37 \\
\hline $\mathrm{P}_{\mathrm{CO}_{2}}$ at admission, median (range), $\mathrm{mm} \mathrm{Hg}$ & $35(31-42)$ & $35(30.75-40)$ & .40 \\
\hline $\mathrm{P}_{\mathrm{aO}_{2}} / \mathrm{F}_{\mathrm{IO}_{2}}$, median (range) & $168(102-266)$ & $128(87-224)$ & .02 \\
\hline Oxygen requirement at admission, median (range), $\mathrm{L} / \mathrm{min}$ & $6(3-11)$ & $12(6-15)$ & $<.001$ \\
\hline No. of lung quadrants with radiographic infiltrates, median (range) & $2(1-4)$ & $2(2-4)$ & $<.001$ \\
\hline \multicolumn{4}{|l|}{ Extrapulmonary organ dysfunction (LODS $>0), n(\%)$} \\
\hline Hemodynamic & $38(29.5)$ & $40(50)$ & .003 \\
\hline Neurological & $5(3.9)$ & $10(12.8)$ & $<.001$ \\
\hline Kidney & $74(58)$ & $58(72.5)$ & .04 \\
\hline Liver & $12(10.3)$ & $18(23.4)$ & .02 \\
\hline Randomization in the group undergoing BAL, $n(\%)$ & $69(53.1)$ & $40(49.4)$ & .67 \\
\hline \multicolumn{4}{|c|}{$\begin{array}{l}\text { Characteristics were compared using the Wilcoxon rank-sum test or the Fisher exact test. } P \text { values }<.05 \text { were then included in the multivariate logistic regression. } \\
\text { LODS = Logistic Organ Dysfunction Score } \\
\text { BAL }=\text { bronchoalveolar lavage }\end{array}$} \\
\hline
\end{tabular}

be noted that after bronchoscopy and BAL, 8 (11\%) subjects who were on oxygen only required NIV institution, and $6(8 \%)$ subjects were intubated.

Three factors were independently associated with invasive mechanical ventilation by multivariate analysis ( $\mathrm{Ta}-$ ble 2): greater oxygen requirements, larger numbers of lung quadrants with radiographic infiltrates, and hemodynamic dysfunction on day 1. In 211 subjects, increased oxygen requirements at admission, increased numbers of lung quadrants involved, and the number of extrapulmonary organ failures were associated with an increased risk of invasive mechanical ventilation (Fig. 3).

The impact of end-of-life decisions on outcomes (withdrawal of life-sustaining treatments) was assessed by multivariate analysis in both intubated and nonintubated subjects. Using these 2 models, determinants of intubation 


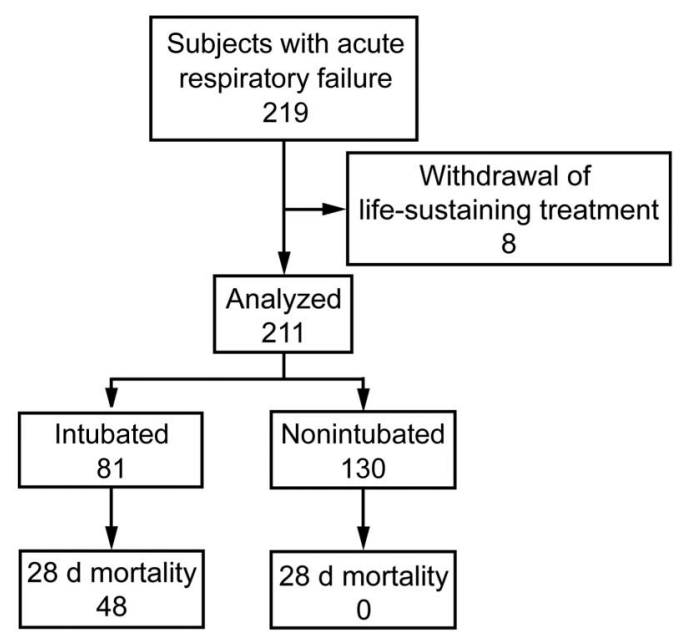

Fig. 1. Subject flow chart and mortality at $28 \mathrm{~d}$.

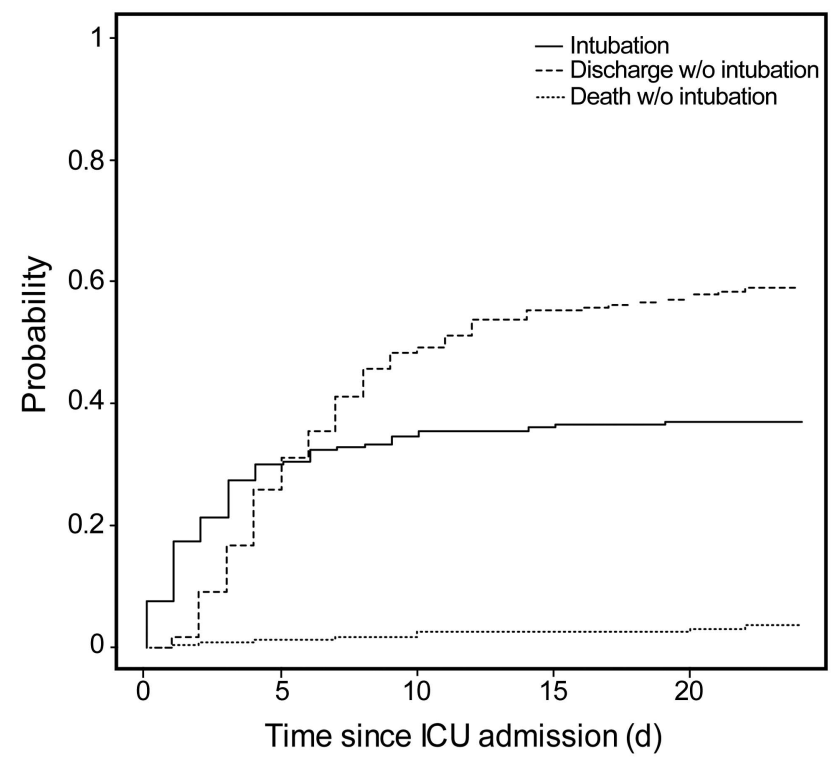

Fig. 2. Probability of intubation according to ICU stay.

were identical with the same independent predictors of intubation.

During the ICU stay, 130 (62\%) subjects required NIV for $>1 \mathrm{~d}$. NIV was successful in $81(62.3 \%)$ subjects, but $49(37.6 \%)$ subjects required second-line invasive mechanical ventilation. ICU mortality for subjects with NIV followed by invasive mechanical ventilation was not different from that for 32 subjects with first-line invasive mechanical ventilation $(65.3 \%$ vs $50 \%, P=.34)$. Risk factors associated with intubation were tested in a subgroup of subjects with NIV failure versus first-line invasive mechanical ventilation. The NIV failure group had higher oxygen requirements on admission $(15[8-15] \mathrm{L} / \mathrm{min}$ vs $9[5-13] \mathrm{L} / \mathrm{min}, P=.02$ ), and other characteristics were
Table 2. Multivariate Analysis of Factors Associated With the Need for Invasive Ventilation During ICU Stay

\begin{tabular}{lrrr}
\hline \hline \multicolumn{1}{c}{ Variable } & OR & 95\% CI & $P$ \\
\hline Oxygen requirement at ICU admission & 1.11 & $1.04-1.19$ & .003 \\
No. of lung quadrants with radiographic & 1.56 & $1.2-2.02$ & .001 \\
$\quad$ infiltrates & & & \\
Extrapulmonary organ dysfunction (LODS) & & & \\
$\quad$ Neurological & 2.45 & $0.66-9.09$ & .18 \\
$\quad$ Hemodynamic & 2.25 & $1.13-4.48$ & .02 \\
$\quad$ Liver & 1.46 & $0.6-3.58$ & .40 \\
$\quad$ kidney & 1.43 & $0.72-2.82$ & .30 \\
ICU center & & & .60 \\
& & & \\
A multivariate logistic regression model was used. & & & \\
OR = odds ratio & & & \\
LODS = Logistic Organ Dysfunction Score & & & \\
\hline
\end{tabular}

not different between the 2 groups (number of involved lung quadrants, extrapulmonary organ dysfunction).

\section{Discussion}

In this study, the risk factors for invasive mechanical ventilation after ICU admission were related to the severity of the respiratory failure and onset of other organ failures. These results are in agreement with previous studies of patients with hematological malignancies. ${ }^{12,29,30}$ The risk factors for invasive mechanical ventilation in our study are objective and easy to assess at the time of ICU admission and maybe even on the wards. Previous studies of patients with hematological malignancies and sepsis or septic shock showed improved outcomes as a result of earlier treatment of organ failures. ${ }^{6,31,32}$ Conceivably, understanding early predictors of invasive mechanical ventilation would improve mortality.

In the present study, subjects receiving NIV were at high risk of invasive mechanical ventilation because $37.6 \%$ of them were eventually intubated. Also, when additional organ failures occurred, the rate of intubation increased (see Fig. 3).

Another striking finding in the present study is that the mortality in subjects who failed NIV was not significantly different from that in first-line intubated subjects. In previous studies, 2,27,29,33 mortality increased after NIV failure and was even higher than mortality after first-line invasive mechanical ventilation. However, these studies reported low survival rates in hematology patients requiring invasive mechanical ventilation. ${ }^{24,29}$ More recently, outcomes have improved.6,31,32 In this study, subjects who received early or prophylactic NIV (ie, NIV trial in a subject without any intubation criteria and no other organ failure) were not distinguished from subjects who received curative NIV (ie, NIV trial in a subject with intubation criteria or asso- 

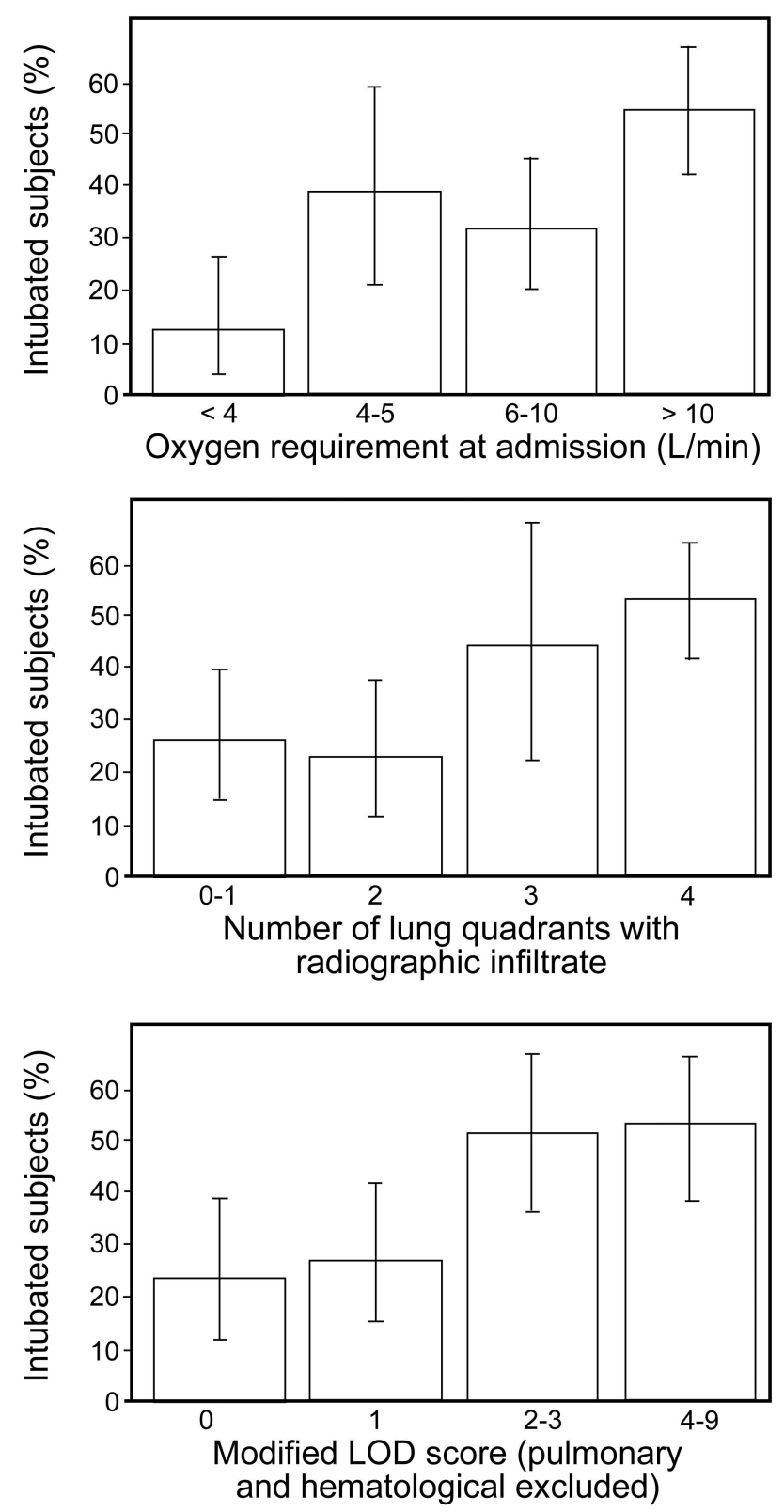

Fig. 3. Risk of intubation at admission according to oxygen requirement at admission, number of lung quadrants with infiltrates on chest x-ray, and number of organ failures (pulmonary and hematological excluded). Whiskers represent $95 \%$ CI. LOD = Logistic Organ Dysfunction.

ciated organ failure). The number of analyzed subjects treated with NIV was limited in this study, so we cannot draw definite conclusions from this set of data. However, subjects in the NIV failure group had greater oxygen requirements at admission but had the same severity score as subjects in the first-line invasive mechanical ventilation group. In a previous study of 99 subjects, ${ }^{27}$ NIV failure was associated with extrapulmonary organ failures.
This study has several limitations. First, we used data from a randomized trial of diagnostic strategies in subjects with malignancies and ARF, and we used this population of nonintubated subjects admitted to the ICU to perform the present study. In theory, the criteria for invasive mechanical ventilation may have varied across study centers. However, the multivariate analysis showed no effect of the center on the invasive mechanical ventilation rate. Failure to avoid intubation in NIV subjects was multifactorial (pulmonary deterioration and/or extrapulmonary organ failure). Second, the study included all subjects with malignancies and ARF due to causes other than cardiogenic edema. Broad ARF etiologies were then included. Outcomes after NIV may be different according to the cause of ARF. ${ }^{34}$ Third, the more severely ill patients were excluded from the analysis, including patients (intubation before ICU or with severe hypoxemia) that may have obvious need for immediate intubation. Moreover, we were not able to analyze a potential bias related to the physician's decision according to the severity of underlying disease or comorbidity. Fourth, ARF and outcomes would be different between solid tumors and hematological malignancy. However, the results of univariate analysis of only hematological subjects were not different (data not shown).

In conclusion, we found that respiratory disease severity and hemodynamic failure at ICU admission were risk factors for invasive mechanical ventilation in subjects with malignancies admitted for ARF. Patients with these risk factors should be considered for invasive mechanical ventilation, particularly when invasive diagnostic procedures would be required. NIV was effective in more than half of the subjects, and NIV failure was associated with a mortality rate similar to first-line invasive mechanical ventilation. The need for invasive mechanical ventilation should be assessed often to ensure that no treatment delay occurs. A prospective study is needed to assess the length and tolerance of NIV before invasive mechanical ventilation.

\section{ACKNOWLEDGMENTS}

We thank A Wolfe for helping to prepare the manuscript.

\section{REFERENCES}

1. Azoulay E, Schlemmer B. Diagnostic strategy in cancer patients with acute respiratory failure. Intensive Care Med 2006;32(6):808-822.

2. Azoulay E, Thiéry G, Chevret S, Moreau D, Darmon M, Bergeron A, et al. The prognosis of acute respiratory failure in critically ill cancer patients. Medicine 2004;83(6):360-370.

3. Chaoui D, Legrand O, Roche N, Cornet M, Lefebvre A, Peffault de Latour R, et al. Incidence and prognostic value of respiratory events in acute leukemia. Leukemia 2004;18(4):670-675.

4. Darmon M, Azoulay E. Critical care management of cancer patients: cause for optimism and need for objectivity. Curr Opin Oncol 2009; 21(4):318-326. 


\section{Identifying Cancer Subjects at Risk for Intubation and Mechanical Ventilation}

5. Azoulay E, Soares M, Darmon M, Benoit D, Pastores S, Afessa B. Intensive care of the cancer patient: recent achievements and remaining challenges. Ann Intensive Care 2011;1(1):5.

6. Pène F, Percheron S, Lemiale V, Viallon V, Claessens YE, Marqué $\mathrm{S}$, et al. Temporal changes in management and outcome of septic shock in patients with malignancies in the intensive care unit. Crit Care Med 2008;36(3):690-696.

7. Ferrà C, Marcos $\mathrm{P}$, Misis M, Morgades M, Bordejé ML, Oriol A, et al. Outcome and prognostic factors in patients with hematologic malignancies admitted to the intensive care unit: a single-center experience. Int J Hematol 2007;85(3):195-202.

8. Groeger JS, Glassman J, Nierman DM, Wallace SK, Price K, Horak D, Landsberg D. Probability of mortality of critically ill cancer patients at $72 \mathrm{~h}$ of intensive care unit (ICU) management. Support Care Cancer 2003;11(11):686-695.

9. Soares M, Salluh JI, Azoulay E. Noninvasive ventilation in patients with malignancies and hypoxemic acute respiratory failure: a still pending question. J Crit Care 2010;25(1):37-38.

10. Lecuyer L, Chevret S, Thiery G, Darmon M, Schlemmer B, Azoulay E. The ICU trial: a new admission policy for cancer patients requiring mechanical ventilation. Crit Care Med 2007;35(3):808-814.

11. Oken MM, Creech RH, Tormey DC, Horton J, Davis TE, McFadden ET, Carbone PP. Toxicity and response criteria of the eastern Cooperative Oncology Group. Am J Clin Oncol 1982;5(6):649-655.

12. Groeger JS, Aurora RN. Intensive care, mechanical ventilation, dialysis, and cardiopulmonary resuscitation. Implications for the patient with cancer. Crit Care Clin 2001;17(3):791-803.

13. Depuydt PO, Benoit DD, Vandewoude KH, Decruyenaere JM, Colardyn FA. Outcome in noninvasively and invasively ventilated hematologic patients with acute respiratory failure. Chest 2004;126(4): 1299-1306.

14. Lecuyer L, Chevret S, Guidet B, Aegerter P, Martel P, Schlemmer B, Azoulay E. Case volume and mortality in haematological patients with acute respiratory failure. Eur Respir J 2008;32(3):748-754.

15. Soares M, Darmon M, Salluh JI, Ferreira CG, Thiéry G, Schlemmer $\mathrm{B}$, et al. Prognosis of lung cancer patients with life-threatening complications. Chest 2007;131(3):840-846.

16. Vincent JL, Akça S, De Mendonça A, Haji-Michael P, Sprung C, Moreno $\mathrm{R}$, et al. The epidemiology of acute respiratory failure in critically ill patients. Chest 2002;121(5):1602-1609.

17. Rabbat A, Chaoui D, Lefebvre A, Roche N, Legrand O, Lorut C, et al. Is BAL useful in patients with acute myeloid leukemia admitted in ICU for severe respiratory complications? Leukemia 2008;22(7): 1361-1367.

18. Azoulay E, Mokart D, Rabbat A, Pene F, Kouatchet A, Bruneel F, et al. Diagnostic bronchoscopy in hematology and oncology patients with acute respiratory failure: prospective multicenter data. Crit Care Med 2008;36(1):100-107.

19. Azoulay E, Mokart D, Lambert J, Lemiale V, Rabbat A, Kouatchet A, et al. Diagnostic strategies in cancer patients with acute respiratory failure: a multicenter randomized controlled trial. Am J Respir Crit Care Med 2010;182(8):1038-1046.
20. Azoulay E, Bergeron A, Chevret S, Bele N, Schlemmer B, Menotti J. Polymerase chain reaction for diagnosing pneumocystis pneumonia in non-HIV immunocompromised patients with pulmonary infiltrates. Chest 2009;135(3):655-661.

21. Azoulay E. Pulmonary infiltrates in patients with malignancies: why and how neutropenia influences clinical reasoning. Eur Respir J 2009;33(1):6-8.

22. Hilbert G, Gruson D, Vargas F, Valentino R, Gbikpi-Benissan G, Dupon $\mathrm{M}$, et al. Noninvasive ventilation in immunosuppressed patients with pulmonary infiltrates, fever, and acute respiratory failure. N Engl J Med 2001;344(7):481-487.

23. Hilbert G, Vargas F, Gruson D. Never the tube! Try the mask! Crit Care Med 2007;35(3):977-978.

24. Hilbert G, Gruson D, Vargas F, Valentino R, Chene G, Boiron JM, et al. Noninvasive continuous positive airway pressure in neutropenic patients with acute respiratory failure requiring intensive care unit admission. Crit Care Med 2000;28(9):3185-3190.

25. Azoulay E, Alberti C, Bornstain C, Leleu G, Moreau D, Recher C, et al. Improved survival in cancer patients requiring mechanical ventilatory support: impact of noninvasive mechanical ventilatory support. Crit Care Med 2001;29(3):519-525.

26. Demoule A, Girou E, Richard JC, Taille S, Brochard L. Benefits and risks of success or failure of noninvasive ventilation. Intensive Care Med 2006;32(11):1756-1765.

27. Adda M, Coquet I, Darmon M, Thiery G, Schlemmer B, Azoulay E. Predictors of noninvasive ventilation failure in patients with hematologic malignancy and acute respiratory failure. Crit Care Med 2008; 36(10):2766-2772.

28. Le Gall JR, Klar J, Lemeshow S, Saulnier F, Alberti C, Artigas A, Teres D. The Logistic Organ Dysfunction system. A new way to assess organ dysfunction in the intensive care unit. ICU Scoring Group. JAMA 1996;276(10):802-810.

29. Antonelli M, Conti G, Moro ML, Esquinas A, Gonzalez-Diaz G, Confalonieri M, et al. Predictors of failure of noninvasive positive pressure ventilation in patients with acute hypoxemic respiratory failure: a multi-center study. Intensive Care Med 2001;27(11):17181728.

30. Soares M, Caruso P, Silva E, Teles JM, Lobo SM, Friedman G, et al. Characteristics and outcomes of patients with cancer requiring admission to intensive care units: a prospective multicenter study. Crit Care Med 2010;38(1):9-15.

31. Pène F, Soares M. Can we still refuse ICU admission of patients with hematological malignancies? Intensive Care Med 2008;34(5):790792.

32. Peigne V, Rusinová K, Karlin L, Darmon M, Fermand JP, Schlemmer B, Azoulay E. Continued survival gains in recent years among critically ill myeloma patients. Intensive Care Med 2009;35(3):512-518.

33. Confalonieri M, Potena A, Carbone G, Porta RD, Tolley EA, Umberto Meduri G. Acute respiratory failure in patients with severe community-acquired pneumonia. Am J Respir Crit Care Med 1999; 160(5 Pt 1):1585-1591.

34. Nava S, Hill N. Non-invasive ventilation in acute respiratory failure. Lancet 2009;374(9685):250-259. 